\title{
The Mind of the Beholder: Luxury Product Placement and Product-Background Scene Congruency: An Abstract
}

\author{
Patricia Rossi, Felipe Pantoja, Kacy Kim, and Sukki Yoon
}

\begin{abstract}
Product placement is a pervasive marketing technique. Focusing on luxury goods, we investigate how luxury associations modulate the effects of productbackground scene congruency on purchase intentions. When a luxury product is placed in a movie scene, it can be visible in a congruent, upscale or incongruent, and downscale background. Past research has investigated plot-connection and product prominence, but less attention has been given to product-background scene congruency. We show that product-background scene congruency leads to higher purchase intentions toward the luxury-placed product only when movie viewers are primed with luxury associations. However, priming participants with non-luxury associations mitigate these effects. Results suggest that simultaneous product-background scene congruency and luxury associations are essential if luxury product placement is to be effective.
\end{abstract}

References Available Upon Request

P. Rossi $(\bowtie)$

IÉSEG School of Management (LEM-CNRS), Lille, France

e-mail: p.rossi@ieseg.fr

F. Pantoja

IÉSEG School of Management, Paris, France

e-mail: f.pantoja@ieseg.fr

K. Kim

Elon University, Elon, NC, USA

e-mail:kkim3@elon.edu

S. Yoon

Bryant University, Smithfield, RI, USA

e-mail: syoon@bryant.edu 